\title{
Malignant glioneuronal tumor of the adult cerebrum with neuropil-like islands involving "proliferating nodules": confirmatory report of an unusual variant
}

\author{
Istvan Vajtai • Michael M. Reinert
}

Received: 1 March 2007 / Revised: 14 March 2007 / Accepted: 14 March 2007 / Published online: 11 April 2007

(C) Springer-Verlag 2007

The focal emergence of subpopulations of tumor cells morphologically displaying some degree of neuronal commitment is being increasingly appreciated as a novel facet to the histological versatility of gliomas of adults [3]. All major histologic subtypes of the latter (i.e. astrocytomas; oligodendrogliomas; ependymomas) have been reported to occasionally involve such differentiation, and the epithet "glioneuronal" rather liberally conferred upon to qualify this phenomenon [7, 10, 12].

Uncommon though they obviously are, reports on detecting neuronal differentiation in gliomas-be it by conventional histology, immunohistochemistry or electron microscopy-do appear with sufficient regularity as for conceptual problems to become manifest. Indeed, diagnostic criteria for purported neuronal differentiation tend to range from expression of neuronal markers in isolated cells to dysplastic neurons, to suggestive architectural patterns $[9,13]$. While the increasing sensitivity of detection methods clearly is at odds with the specific relevance of results thus obtained, reproducible patterns are being searched for in order for meaningful entities to be singled out.

Introduced by Teo et al. in 1999, the so-called "glioneuronal tumor with neuropil-like islands" has subsequently been the subject of a handful of case studies confirming a remarkably constant clinicopathologic presentation of this neoplasm [3 and references therein]. This includes occur-

\section{Vajtai $(\square)$}

Section of Neuropathology, Institute of Pathology,

University of Bern, Murtenstrasse 31, Postfach 62,

3010 Bern, Switzerland

e-mail: istvanvajtai@yahoo.com

M. M. Reinert

Department of Neurosurgery,

Inselspital, Bern, Switzerland rence in the cerebral hemispheres of adults, involving an infiltrating astrocytoma (WHO grade II or higher) punctuated by discreet clusters of round cells enmeshed within a Synaptophysin-immunoreactive feltwork. With but one exception - the case documented by Keyvani et al.- the cells engaged in the neuropil-like islands have been felt not to represent the most intensely proliferating moiety of the lesions [5]. Although the mere presence of neuropil-like islands does not seem to bestow a particularly favorable prognosis, the modest proliferative activity of these is likely to be intuited to connote maturation.

We recently had the privilege of studying a high-grade glioma with intensely proliferating neuropil islands surgically removed from the left frontal lobe of a 59-year-old woman. By World Health Organization criteria, the major part of the tumor (approximately 70\%) corresponded to conventional glioblastoma (WHO grade IV), i.e. an astrocytoma with cellular anaplasia, mitotic figures and microvascular proliferation as well as extensive palisading necrosis (Fig. 1a, b). In addition, several discreet aggregates of nondescript round cells with inconspicuous cytoplasm and round darkly stained, primitive-appearing nuclei were encountered (Fig. 1c). Measuring 40-350 $\mu \mathrm{m}$ in diameter, the contours of each of these variably appeared as smudged to rather clear-cut (Fig. 1d, f). Within individual clusters, cells either were interwoven with delicate fibrillary processes, or tended to haphazardly collapse to produce nuclear crowding. Irrespective of architecture, such foci stained intensely and almost exclusively for Synaptophysin as opposed to surrounding GFAP-positive astrocytes (Fig. 1e, $\mathrm{g}, \mathrm{h})$. No gangliocytic maturation or any suggestive immunoreactivity for neurofilament proteins was found. While MIB-1 counts averaged some $9-11 \%$ in the intervening astrocytic tumor component, nuclear labeling within the neuropil-like nodules was as high as $40 \%$ (Fig. 1i). 

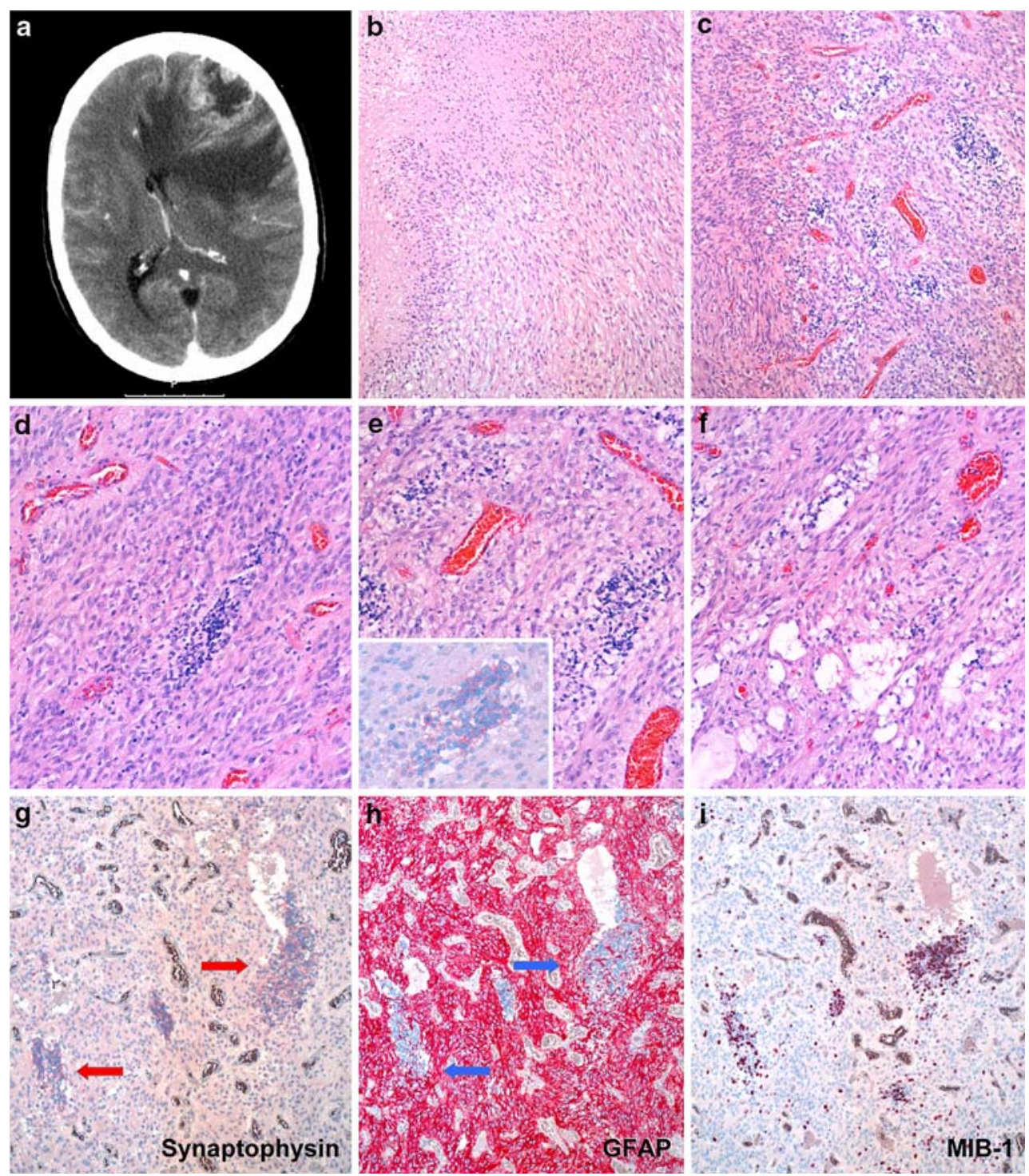

Fig. 1 Postcontrast axial computed tomography (a) to show superficial, centrally necrotic tumor mass of $4.6 \mathrm{~cm}$ diameter near left frontal pole. Intense ring enhancement and marked perifocal edema are noted. Histologically, a high-grade astrocytic neoplasm with palisading necroses (b) corresponding to glioblastoma (WHO grade IV) accounts for the main bulk of the lesion. Focally (c), the gliomatous texture is disrupted by clusters of hyperchromatic round cells with cytoplasmic clearing. Architectural variations of such "neuropil-like islands" range from ill-defined condensations of nuclei (d), to fibrillated nodules (e) to clumps of haphazardly collapsed cells along the wall of irregular

The histologic aspect of the present case closely-if not entirely - parallels the findings by Keyvani et al., in particular as it is felt to represent the first confirmatory report of a glioneuronal tumor with neuropil-like islands involving proliferating nodules [5]. In keeping with previous descriptions of hybrid lesions of a similar ilk, the term "neuronal"-as it refers to nodules-is meant to denote but an immunophenotype compatible with elementary neurosecretion in tumor cells that do not express glial fibrillary acidic protein. vacuoles (f). Inset in e illustrates Synaptophysin immunoreactivity in neuropil-like islands. On consecutive section planes, reciprocal staining patterns for Synaptophysin (g) and GFAP (h) assist in identifiying neuropil-like islands (arrows) and astrocytic tumor background, respectively. Intense nuclear labeling for MIB-1 is appreciated in "proliferating nodules" (i), especially as opposed to surrounding glioma tissue. Microphotographs not labeled otherwise represent hematoxylineosin staining. Original magnification, $\mathbf{b}-\mathbf{c}$ and $\mathbf{h}-\mathbf{i} \times 100$; d-f $\times 200$; inset in $\mathbf{e} \times 400$

Prior to the observation by Keyvani et al.- -and indeed antedating the concept of "neuropil-like islands"actively proliferating neuronal cells within composite glioneuronal neoplasms had variously been interpreted as either malignant ganglioglioma or primitive neuroectodermal tumor. To cite but one of the more recent contributions of this type, McLendon et al. described two such examples of cerebral neuroblastoma associated with anaplastic astrocytoma and glioblastoma, respectively [6]. 
While cytologically and immunophenotypically comparable to the cells involved in the "neuropil-like islands" of our case, the neuroblastic moiety observed by these authors was either characterized by a "remarkable profusion of small cells" (case 1) or felt to qualify as neuroblastoma of "classical type" (case 2) complete with Homer Wright rosettes. Moreover, at variance with the focal distribution and altogether minoritary character of the primitive neuronal population in the present case, the small cell neuronal component therein did predominate in both lesions.

Of late, Shibahara et al. observed emergence of a neuronal immunophenotype during progression of an astrocytoma to glioblastoma, the latter also including a small cell component [11]. While "small numbers of tumor cells that were hard to discriminate on hematoxylin-eosin stain" did also express neuronal markers in the low-grade precursor lesion, these were not felt to clearly segregate in clusters. Likewise, immunoreactivity for Synaptophysin and NeuN tended to occur "diffusely", rather than in discreet nodules, after malignant change has taken place.

Currently, the nature of the biologic process reflected by neuropil-like islands only allows for tentative interpretation, one partly drawing on perceived similarities with known patterns of heterologous differentiation in gliomas. On the one hand, neuropil-like islands can be conceived as transitory structures that may eventually either undergo definite gangliocytic maturation - thus evolving into complex glioneuronal tumors of the type described by Rodriguez et al. [9] - or end up submerged by the mitotically active astrocytic bulk of tumor. This hypothetic scenario has actually been lent some support by cases requiring second surgery, in which the neuronal component was reported as either not being present from the outset [8] or vanishing during tumor progression [12]. Conversely, cells within neuropil-like islands are apt to invite reading-by analogy_as ones reminiscent of embryonal CNS neoplasia, especially PNET and medulloblastoma. Of note, sporadic mentions of glioblastoma admixed with a poorly differentiated PNET-like component are indeed on record [4]. Moreover, either spontaneous or therapy-related "maturation" of medulloblastoma involving neuronal as well as glial lineages, has been described $[1,2]$.

Lately, pluripotent precursor cells participating in adult neurogenesis have been identified, and indeed put forward as potential targets of neoplastic transformation [14]. While evidence in support of the concept of adult CNS stem cell neoplasia is being gathered, it seems allowable to speculate that glioneuronal tumors with neuropil-like islands may possibly derive from such pathomechanism. The identification of both mitotically inactive and proliferating variants of neuropil-like islands, as the one documented here, argues for these to be dynamic structures-even allowing for their presence to be conceived as ephemeral (therefore contingent on sampling). In either form, these possibly represent a morphologically defined compartment of cells actively involved in the clonal evolution of gliomas.

\section{References}

1. Ammar A, Al-Majid H, Anim JT, Kutty MK, Ibrahim AW (1991) Differentiating medulloblastoma in adults. Neurol Res 13:125127

2. Cai DX, Mafra M, Schmidt RE, Scheithauer BW, Park TS, Perry A (2000) Medulloblastomas with extensive posttherapy neuronal maturation. Report of two cases. J Neurosurg 93:330-334

3. Edgar MA, Rosenblum MK (2007) Mixed glioneuronal tumors. Recently described entities. Arch Pathol Lab Med 131:228-233

4. Ishizawa K, Kan-nuki S, Kumagai H, Komori T, Hirose T (2002) Lipomatous primitive neuroectodermal tumor with a glioblastoma component: a case report. Acta Neuropathol 103:193-198

5. Keyvani K, Rickert CH, von Wild K, Paulus W (2001) Rosetted glioneuronal tumor: a case with proliferating neuronal nodules. Acta Neuropathol 101:525-528

6. McLendon RE, Bentley RC, Parisi JE, Tien RD, Harrison JC, Tarbell NJ, Billitt AL, Gualtieri RJ, Friedman HS (1997) Malignant supratentorial glial-neuronal neoplasms. Report of two cases and review of the literature. Arch Pathol Lab Med 121:485-492

7. Perry A, Scheithauer BW, Macaulay RJB, Raffel C, Roth KA, Kros JM (2002) Oligodendrogliomas wit neurocytic differentiation. A report of 4 cases with diagnostic and histogenetic implications. J Neuropathol Exp Neurol 61:947-955

8. Prayson RA, Abramovich CM (2000) Glioneuronal tumor with neuropil-like islands. Hum Pathol 31:1435-1438

9. Rodriguez FJ, Scheithauer BW, Port JD (2006) Unusual malignant glioneuronal tumors of the cerebrum of adults: a clinicopathologic study of three cases. Acta Neuropathol 112:727-737

10. Rodriguez FJ, Scheithauer BW, Robbins PD, Burger PC, Hessler RB, Perry A, Abell-Aleff PC, Mierau GW (2007) Ependymomas with neuronal differentiation: a morphologic and immunohistochemical spectrum. Acta Neuropathol 113:313-324

11. Shibahara J, Fukayama M (2005) Secondary glioblastoma with advanced neuronal immunophenotype. Virchows Arch 447:665668

12. Teo J, Gultekin SH, Bilsky M, Gutin P, Rosenblum MK (1999) A distinctive glioneuronal tumor of the adult cerebrum with neuropil-like (including "rosetted") islands: Report of 4 cases. Am J Surg Pathol 23:502-510

13. Varlet P, Soni D, Miquel C, Roux FX, Meder JF, Chneiweiss H, Daumas-Duport C (2004) New variants of malignant glioneuronal tumors: a clinicopathological study of 40 cases. Neurosurgery 55:1377-1392

14. Vescovi AL, Galli R, Reynolds BA (2006) Brain tumour stem cells. Nat Rev Cancer 6:425-436 